\title{
A Novel Smoking Cessation Smartphone App Integrated With a Mobile Carbon Monoxide Checker for Smoking Cessation Treatment: Protocol for a Randomized Controlled Trial
}

Akihiro Nomura ${ }^{1,2,3}, \mathrm{MD}, \mathrm{PhD}$; Hiroki Tateno ${ }^{4,5}, \mathrm{MD}, \mathrm{PhD}$; Katsunori Masaki ${ }^{4}, \mathrm{MD}, \mathrm{PhD}$; Tomoyasu Muto ${ }^{6}, \mathrm{MSc}$; Shin Suzuki ${ }^{6}$, MD; Kohta Satake ${ }^{1,6}$, MD, MBA, MPH; Eisuke Hida ${ }^{7}$, PhD; Koichi Fukunaga ${ }^{4}$, MD, PhD

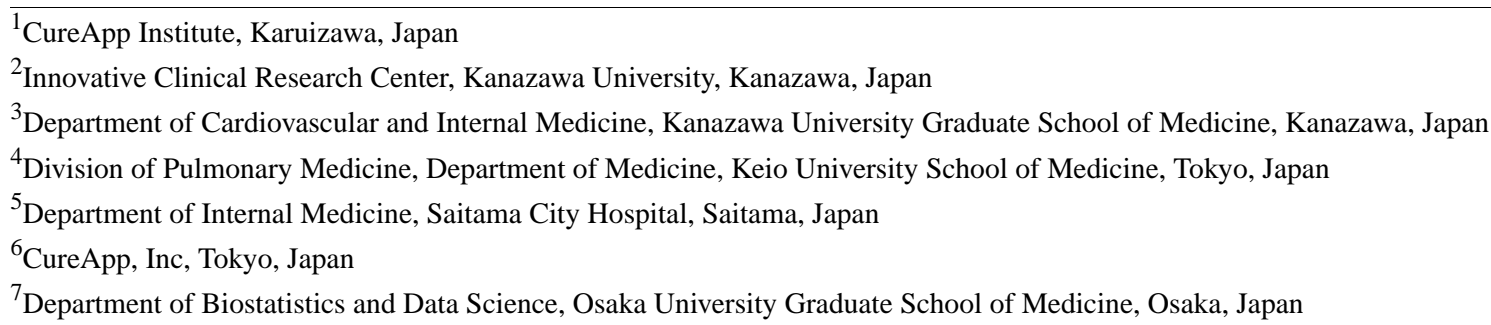

\section{Corresponding Author:}

Hiroki Tateno, MD, PhD

Department of Internal Medicine

Saitama City Hospital

2460 Mimuro, Midori-ward

Saitama, 3368522

Japan

Phone: 81488734111

Email: hrk12tateno@me.com

\section{Abstract}

Background: Smoking cessation treatment programs have been widely available for patients with nicotine dependence. Despite intensive programs, the continuous abstinence rate (CAR) from weeks 9-12 is still about 50\%. Recently, a smartphone app emerged as a novel tool for therapeutic interventions, including nicotine dependence. In this study, we developed "CureApp Smoking Cessation" (CASC), which consists of a smartphone app for patients and a Web-based patient management software for doctors with a mobile carbon monoxide (CO) checking device to improve the efficacy of the smoking cessation treatment.

Objective: This study aims to evaluate whether the CASC app is effective for individuals with nicotine dependence in addition to standard smoking cessation programs.

Methods: This will be a randomized, sham-controlled, open-label, multicenter trial. We will recruit participants with nicotine dependence, but are otherwise healthy adults. We will randomize and allocate participants 1:1 to the CASC treatment group or a control app group. Both groups will receive a 12-week standard smoking cessation program with pharmacotherapy and counseling. In addition, participants in the treatment group will have the CASC app installed on their smartphone, which will provide video tutorials, advice from an artificial intelligence nurse, a digital diary, and measure daily exhaled CO concentration. In contrast, the control group will have the control app installed on their smartphone, where all the functions that can potentially effect smoking cessation are removed. The primary outcome will be the biochemically validated CAR from weeks 9-24. The success of smoking cessation will be defined as self-reported continuous abstinence from weeks 9-24 and exhaled CO concentration $\leq 10$ ppm both at weeks 12 and 24. The main secondary outcomes will be the CAR from weeks 9-12, weeks 9-52, and 7-day point prevalence abstinence at weeks $4,8,12,24$, and 52.

Results: We will recruit 580 participants with nicotine dependence from October 2017 to September 2018 or until the recruitment process is complete. The final 52-week follow-up will be completed in October 2019. We expect all trial results to be available by the end of 2019. The trial is funded by CureApp, Inc.

Conclusions: This is the first randomized controlled trial to evaluate the efficacy of CASC. We expect that CASC, in addition to standard smoking cessation programs, has a significantly higher CAR during weeks 9-24 than the control app. 
Trial Registration: University Hospital Medical Information Network Clinical Trials Registry UMIN000031589; https://upload.umin.ac.jp/cgi-open-bin/ctr/ctr_view.cgi?recptno=R000033555

International Registered Report Identifier (IRRID): DERR1-10.2196/12252

(JMIR Res Protoc 2019;8(2):e12252) doi: 10.2196/12252

\section{KEYWORDS}

continuous abstinence rate; nicotine dependence; telemedicine; randomized controlled trial; smoking cessation; tobacco use disorder

\section{Introduction}

Smoking is a risk factor that causes various diseases such as malignant tumors, heart disease, cerebrovascular disease, and chronic obstructive pulmonary disease [1]. It has been estimated that there are 22 million (approximately $18 \%$ of the total Japanese population) of adult smokers in Japan, and the number of deaths attributable to smoking is approximately 129,000 per year, indicating that smoking is the most common extrinsic cause of death among noninfectious diseases [2]. In addition, excess medical expenses owing to smoking are up to 1.5 trillion yen (US $\$ 13$ billion) [3]. Therefore, reducing smoking prevalence could contribute to not only prevent the onset of these lifestyle-related diseases and cancer but also decrease future medical costs [4].

Smoking cessation treatment programs have been widely available for patients with nicotine dependence supported by the Japanese national insurance system [5]. This program consists of 5 visits spanning 12 weeks providing counseling by health care professionals and pharmacotherapy, including nicotine patch and varenicline. Despite this intensive treatment program, the continuous abstinence rate (CAR) from weeks 9-12 has still been 40\%-65\% [6-8]. Moreover, it significantly decreases after the program finishes and is around $40 \%$ at 1-year follow-up [7]. To improve both in-program and long-term CAR, we need additional and more effective treatment interventions both during the regular outpatient clinic visits and after the completion of the 12-week program [6].

Medical apps for smartphones are emerging as "therapeutic" intervention tools to ameliorate diabetes mellitus $[9,10]$, depression [11], and nicotine dependence [12,13]. Because they cover interoutpatient visit blank periods (ie, intervals between clinic visits during which patients normally cannot obtain any clinical guidance from medical staffs), these apps are useful to continuously monitor, promote, and encourage the treatment program from medical staff, as well as by patients themselves. In this regard, Free et al [13] reported that smoking cessation support with daily or weekly text messages during the program doubled smoking quit rates. Although smartphone apps for nicotine dependence are already available in the United States, it remains unclear whether the app with a mobile carbon monoxide (CO) checker device is safe and effective in patients with nicotine dependence.

Recently, we developed "CureApp Smoking Cessation" (CASC), which consists of the latest version of CureApp
Smoking Cessation smartphone app for patients and a Web-based patient management software for primary physicians with a mobile $\mathrm{CO}$ checking device to improve the success of the smoking cessation treatment. The CASC provides users with accurate knowledge of nicotine dependence, clues to change their behaviors, and measurements of their own exhaled $\mathrm{CO}$ concentration levels at home. In addition, patients can share these data remotely with their primary physicians. Masaki et al reported that, in the previous prospective studies with participants using prototypes of CASC smartphone app without a mobile $\mathrm{CO}$ checker, the results showed the feasibility and usability in the phase I study [14] and demonstrated a higher CAR from weeks 9-24 than the national surveys in the phase II study [15].

Therefore, this study aims to evaluate whether the latest version of CASC in addition to a standard smoking cessation program is effective in treating individuals with nicotine dependence.

\section{Methods}

\section{Overall Trial Design}

Figure 1 shows the flowchart of the trial. This trial will be a randomized, sham-controlled, open-label, multicenter trial. Table 1 shows trial centers. This trial aims to assess the efficacy of CASC in patients with nicotine dependence when added to a standard smoking cessation program. The treatment group will use the CASC smartphone app integrated with a mobile $\mathrm{CO}$ checker and a Web-based patient management software for 24 weeks. Table 2 shows the overall follow-up schedule. The primary outcome of this study will be the CAR from weeks 9-24. We hypothesize that the CASC treatment group will have a higher CAR than the control app group from weeks 9-24. The CAR is defined as the percentage of individuals continuously not smoking even a puff during the specified period. We will conduct this trial in compliance with the Declaration of Helsinki, Medical Device Good Clinical Practice, Ethical Guidelines for Medical and Health Research Involving Human Subjects, and all other applicable laws and guidelines in Japan. This protocol and informed consent forms were approved by the Institutional Review Board at Keio University School of Medicine and affiliated institutions. Furthermore, we will use the latest version of the approved documents. This study is registered at the University Hospital Medical Information Network Clinical Trials Registry (UMIN000031589). 
Figure 1. Flowchart of the trial protocol. CASC: CureApp Smoking Cessation.

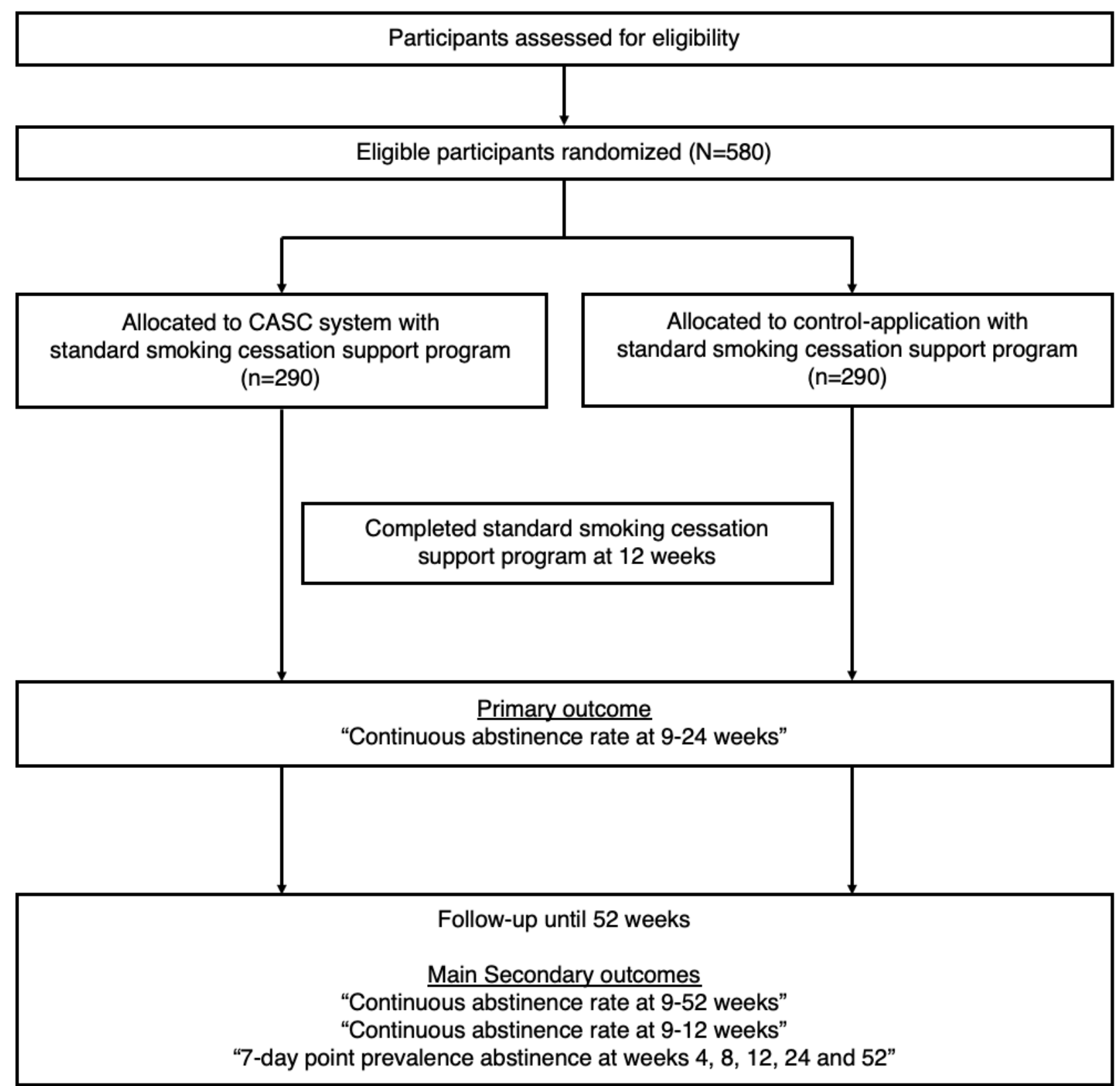

\section{Trial Participants}

We will recruit nicotine-dependent adult participants from October 2017 to September 2018 or until the recruitment process completes; we plan to follow them until October 2019. Participants who meet all inclusion criteria will be included in the trial and those fulfilling the exclusion criteria will be removed from this trial (Textbox 1). We will obtain written informed consent from all trial participants. Participants should understand the content of the consent form sufficiently before their acceptance. Consent forms must be dated and signed both by trial participants and researchers. When consent is obtained, the first copy of the form will be kept in each institution, while the other part will be kept by participants and will not be collected even after the trial is over. In addition, we will inform all participants that their medical care will not be affected if they refuse to enroll in this trial. Participants will be able to leave the trial whenever they want to discontinue it. All participants will receive a compensation of 10,000 yen per clinic visit (7 visits in total).

\section{Randomization}

Randomization will be performed for eligible participants. They will be allocated 1:1 to both arms-the CASC treatment group and the control app group. Randomization will be performed by a computer-generated random sequence with stratification for smoking cessation medications. 
Table 1. The list of trial centers.

\begin{tabular}{|c|c|c|}
\hline Number & Institution & Prefecture \\
\hline 1 & Ankou Medical Clinic & Tokyo \\
\hline 2 & Ayano Clinic & Saitama \\
\hline 3 & BOOCS Clinic Fukuoka & Fukuoka \\
\hline 4 & Chuo Naika Clinic & Tokyo \\
\hline 5 & Ebisu Garden Place Clinic & Tokyo \\
\hline 6 & Higashi-hie Naika & Fukuoka \\
\hline 7 & Hosoda Shinryojo (clinic) & Tokyo \\
\hline 8 & Inoue Naika Clinic & Ibaraki \\
\hline 9 & Kanda Clinic & Tokyo \\
\hline 10 & Keio University Hospital & Tokyo \\
\hline 11 & Kimura-Shiro Clinic & Fukuoka \\
\hline 12 & Kita Shin-Yokohama Naika Clinic & Kanagawa \\
\hline 13 & Mashiba Iin (clinic) & Saitama \\
\hline 14 & Maekawa Medical Clinic & Kanagawa \\
\hline 15 & Miyazaki RC Clinic & Tokyo \\
\hline 16 & Motosumiyoshi Kokoromi Clinic & Kanagawa \\
\hline 17 & Nakameguro Atlas Clinic & Tokyo \\
\hline 18 & National Center for Global Health and Medicine & Tokyo \\
\hline 19 & Nemoto Geka-Seikeigeka (clinic) & Saitama \\
\hline 20 & Nihonbashi Naika-Allegy Clinic & Tokyo \\
\hline 21 & Nomura Iin (clinic) & Tokyo \\
\hline 22 & Odayaka Life Naika Clinic & Saitama \\
\hline 23 & Saitama City Hospital & Saitama \\
\hline 24 & Sawayama Naika-Sougoushinryou Clinic & Fukuoka \\
\hline 25 & Segawa Hospital & Saitama \\
\hline 26 & Shimizu Clinic Fusa & Saitama \\
\hline 27 & Shinjuku Research Park Clinic & Tokyo \\
\hline 28 & Sone Clinic Shinjuku & Tokyo \\
\hline 29 & Tajima Clinic & Tokyo \\
\hline 30 & Tenjin Sogo Clinic & Fukuoka \\
\hline 31 & Ueda Naika Clinic & Fukuoka \\
\hline
\end{tabular}

\section{Intervention and Control}

In addition to the 12-week standard treatment procedure for smoking cessation [5], we will prescribe the CASC to the treatment group. On the other hand, a control app will be prescribed to the control group. The standard 12-week smoking cessation treatment protocol provides 5 on-site examinations and in-person counseling by a primary physician at each outpatient clinic within 3 months.

CASC consists of a CureApp Smoking Cessation smartphone app based on a cloud system paired with a mobile $\mathrm{CO}$ checker for participants and a Web-based patient management software for primary care physicians. Participants in the treatment group will install the app in their smartphones and then begin taking a few minutes every day for watching a video tutorial regarding smoking cessation; in addition, participants from the treatment group will keep a digital diary of quitting smoking, chat with artificial intelligence (AI) nurse, and check their exhaled $\mathrm{CO}$ concentration by the mobile $\mathrm{CO}$ checker at least once a day. Physicians will be able to follow participants' exhaled CO concentration data and physical conditions through a secure cloud system and review them during the clinic visits (Figure 2). 
Table 2. The trail assessment and evaluation schedule.

\begin{tabular}{|c|c|c|c|c|c|c|c|c|}
\hline \multirow[t]{2}{*}{ Assessments } & \multicolumn{7}{|c|}{ Observational period } & \multirow[t]{2}{*}{ At withdrawal } \\
\hline & $\begin{array}{l}\text { Day of registra- } \\
\text { tion (Day 1) }\end{array}$ & $\begin{array}{l}2 \text { weeks } \\
\text { (Day 15) }\end{array}$ & $\begin{array}{l}4 \text { weeks } \\
\text { (Day 29) }\end{array}$ & $\begin{array}{l}8 \text { weeks } \\
\text { (Day 57) }\end{array}$ & $\begin{array}{l}12 \text { weeks } \\
\text { (Day } 85)\end{array}$ & $\begin{array}{l}24 \text { weeks } \\
\text { (Day 169) }\end{array}$ & $\begin{array}{l}52 \text { weeks } \\
\text { (Day 366) }\end{array}$ & \\
\hline Patients' background & $\checkmark$ & & & & & & & \\
\hline $\begin{array}{l}\text { Tobacco Dependence } \\
\text { Screener }\end{array}$ & $\checkmark$ & & & & & & & \\
\hline Brinkman Index ${ }^{a}$ & $\checkmark$ & & & & & & & \\
\hline $\begin{array}{l}\text { Fagaström Test for Nicotine } \\
\text { Dependence }\end{array}$ & $\checkmark$ & & & & & & & \\
\hline $\begin{array}{l}\text { 12-item French version of } \\
\text { the Tobacco Craving Ques- } \\
\text { tionnaire }\end{array}$ & $\checkmark$ & $\checkmark$ & $\checkmark$ & $\checkmark$ & $\checkmark$ & $\checkmark$ & $\checkmark$ & \\
\hline $\begin{array}{l}\text { Kano Test for Social Nico- } \\
\text { tine Dependence }\end{array}$ & $\checkmark$ & & & $\checkmark$ & $\checkmark$ & $\checkmark$ & $\checkmark$ & \\
\hline $\begin{array}{l}\text { Mood and Physical Symp- } \\
\text { toms Scale }\end{array}$ & $\checkmark$ & $\checkmark$ & $\checkmark$ & $\checkmark$ & $\checkmark$ & $\checkmark$ & $\checkmark$ & \\
\hline $\begin{array}{l}\text { Exhaled carbon monoxide } \\
\text { concentration }\end{array}$ & $\checkmark$ & $\checkmark$ & $\checkmark$ & $\checkmark$ & $\checkmark$ & $\checkmark$ & $\checkmark$ & $\checkmark$ \\
\hline Status of nonsmoking & $\checkmark$ & $\checkmark$ & $\checkmark$ & $\checkmark$ & $\checkmark$ & $\checkmark$ & $\checkmark$ & $\checkmark$ \\
\hline Status of device use & & $\checkmark$ & $\checkmark$ & $\checkmark$ & $\checkmark$ & $\checkmark$ & & $\checkmark$ \\
\hline Adverse events & $\checkmark$ & $\checkmark$ & $\checkmark$ & $\checkmark$ & $\checkmark$ & $\checkmark$ & $\checkmark$ & $\checkmark$ \\
\hline
\end{tabular}

${ }^{\mathrm{a}}$ Brinkman index $=($ number of cigarettes per day $) \times($ number of smoking years $)$.

Textbox 1. Inclusion and exclusion criteria.

Inclusion:

- Participants diagnosed with nicotine dependence (Tobacco Dependence Screener $\geq 5$ points)

- Participants with Brinkman index $\geq 200$

- Participants who wish to quit smoking immediately

- Participants who agree to receive the smoking cessation treatment program and sign the written consent form

- Participants who can use a smartphone (operating system: Android 5.0 and above or iPhone 8.0 and above)

Exclusion:

- $\quad$ Participants with severe mental illness

- Participants who cannot come to their follow-up clinic visits during the 1 year

- Participants who have started taking a smoking cessation medication within 1 year before the registration

- Participants who plan to use any smoking cessation aids and participate in any kind of smoking cessation activities (not limited to smoking cessation therapy) outside the trial

- Participants whose investigator or clinical trial doctor judged them to be unsuitable for participation in this trial owing to other reasons

On the other hand, participants from the control app group will be able to perform only 6 basic functions in their app as follows: (1) show the user guide (how to use the app); (2) enter their profile and set the start date of smoking cessation; (3) display the course of 5 visits during the 12-week treatment with a summary of objectives of each visit; (4) show the date of next appointment; (5) get the contact form for technical support; and
(6) display an app version, privacy policy, and other administrative information. The other functions of the CASC app will not be incorporated in the control app. The control app will not include the mobile $\mathrm{CO}$ checker either. Note that exhaled $\mathrm{CO}$ concentrations will be measured by the medical staff at each clinic visit regardless of the study groups, and these $\mathrm{CO}$ data at the clinic will be used in the analysis of study outcomes. 
Figure 2. Overview of the "CureApp Smoking Cessation". CO: carbon monoxide; IoT: Internet of Things.

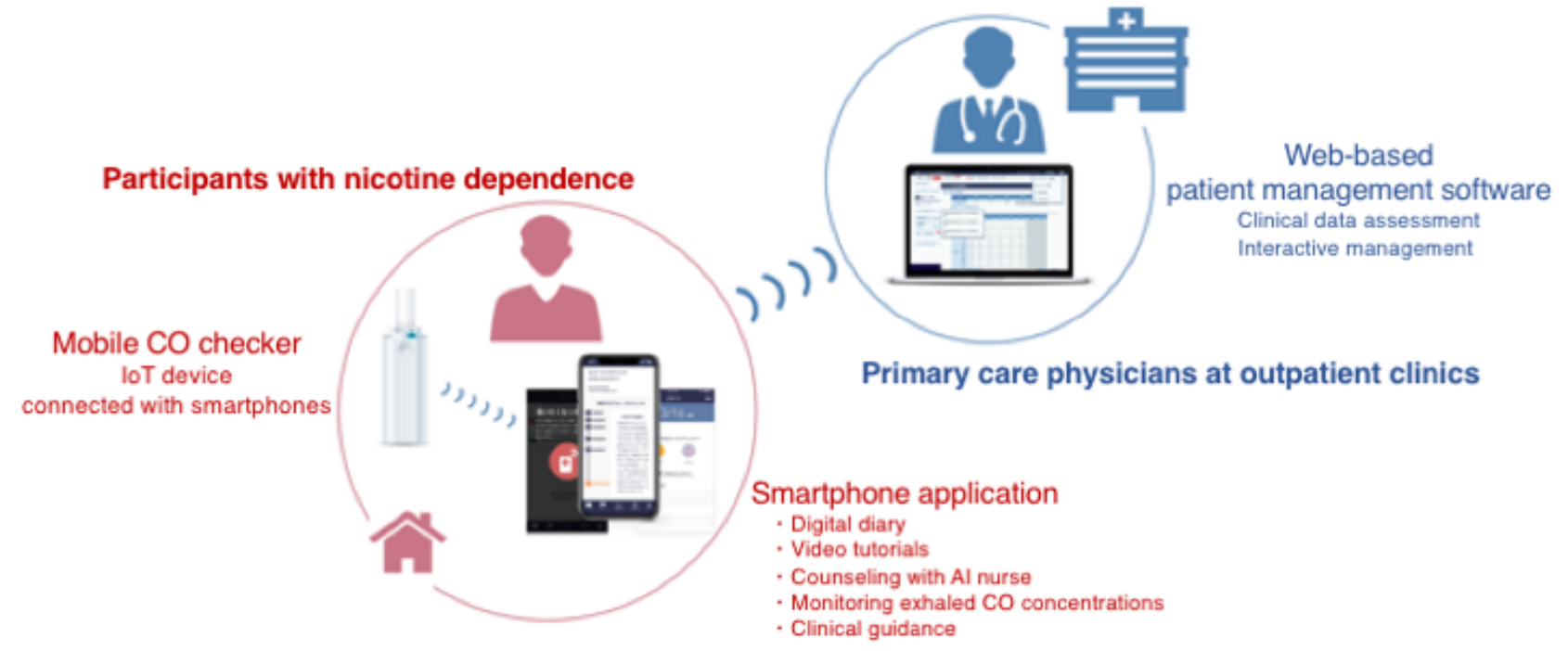

The CASC, control app, and mobile CO checker delivered by CureApp Inc. (Tokyo, Japan) should be carefully managed to ensure they are not used outside the clinical trial at each medical institution. A prescription code will be required to activate the investigational device, and the control group app will be issued by sponsors specific to each practicing medical institution; these sponsors regularly (at least once a year) perform inventory confirming there will be no usage of the app outside the trial.

\section{Overview of the "CureApp Smoking Cessation" Smartphone app}

The CASC smartphone app was developed at CureApp Inc. supervised by the Division of Pulmonary Medicine, Department of Medicine, Keio University School of Medicine. The app runs on both iOS and Android smartphones. Primary physicians will provide the app prescription code to participants at their outpatient clinics. Participants will download the app through their smartphones, activate the app entering the code, and enter their baseline data including age, sex, years of smoking, number of cigarettes per day, prescribed medications (nicotine patch or varenicline), and motivation and self-confidence regarding smoking cessation. These data will be securely sent to the cloud storage, and the AI of our system will assemble tailor-made smoking cessation programs suitable to each participant based on their personal information. Personal data stored on the cloud can be reviewed by primary physicians. The CASC app has the following 4 main components to maximize the therapeutic effects of pharmacological therapy and counseling provided by health care professionals: (1) keeping a smoking cessation diary (once a day); (2) lectures and educational videos helping its users quit smoking; (3) interactive counseling by chat-bot (AI nurse); and (4) daily measurement and recording of exhaled $\mathrm{CO}$ concentration levels at home through a mobile $\mathrm{CO}$ checker.

Regarding a Web-based personal computer software for primary doctors, it provides a data management app from patients' CASC mobile apps and advice for physicians to follow the national clinical guidelines appropriately.

\section{Outcomes}

The primary outcome of this study will be the biochemically validated CAR from weeks 9-24. The success of smoking cessation has been defined as (1) self-reported continuous abstinence from weeks 9-24 and (2) exhaled CO concentration no more than $10 \mathrm{ppm}$ at both weeks 12 and 24. In current smoking cessation programs, the CAR from weeks 13-24 drastically decreases, whereas our investigational device is expected to prevent this reduction by covering "treatment gaps" to promote the retention of appropriate recognition and behavioral changes. A single-arm prospective pilot study showed that a CASC app, even without a mobile CO checker, had a significantly higher CAR from weeks 9-24 than the historical cohort that had received standard smoking cessation program $[16,17]$.

In addition, we will evaluate the following secondary outcomes: (1) 7-day point prevalence abstinence at weeks 4, 8, 12, 24, and 52; (2) the CAR from weeks 9-12 and from weeks 9-52; (3) changes in several scores related to smoking cessation, including the Mood and Physical Symptoms Scale (MPSS) [18], 12-item French version of the Tobacco Craving Questionnaire translated into Japanese (FTCQ-12) [19], Kano Test for Social Nicotine Dependence (KTSND) [20], and time to first lapse after initial visit; (4) usage rates of smartphone apps; and (5) the presence of product problems or adverse events.

\section{Follow-Up Schedule}

Table 2 shows the overall follow-up schedule of this trial. At registration, patients' background profile, exhaled $\mathrm{CO}$ concentration, nicotine dependence, and smoking status will be assessed using instruments such as the Tobacco Dependence Screener [21], Brinkman index (multiplied by cigarettes per day and years of smoking), Fagerström Test for Nicotine Dependence (FTND), FTCQ-12, KTSND, and MPSS. The background profile includes age, sex, body weight, years of smoking, cigarettes per day, and medical history at the initiation of device use. Regular follow-up visits will be scheduled at 2 weeks ( \pm 7 days), 4 weeks ( \pm 7 days), 8 weeks ( \pm 14 days), 12 weeks ( \pm 14 days), 24 weeks ( \pm 28 days), and 52 weeks $( \pm 28$ days); during these follow-up visits, we will examine patients' 
scores regarding FTCQ-12, KTSND (after 8 weeks), and MPSS, as well as exhaled $\mathrm{CO}$ concentration, status of nonsmoking, status of device use, and adverse events. Adverse events and concomitant pharmacological therapy will be recorded through the trial.

An independent monitoring staff will conduct on-site data monitoring. In addition, the staff will review trial database to confirm whether principal and subinvestigators at each clinic perform the clinical trial according to the research proposal and standard operation procedures, and data queries will be asked if necessary.

\section{Participant Withdrawal Criteria}

Participants will be discontinued from follow-up visits for this trial when they meet either of the following criteria: (1) participants request discontinuation; (2) researchers consider discontinuation is necessary owing to severe adverse effects, for example; (3) the eligibility criteria was violated; (4) significant deviation from the protocol occurred; (5) study institution terminated trial participation because of good clinical practice violation, for example; (6) participants' further follow-up visits are considered very difficult to accomplish; (7) continuing smoking even at the $9^{\text {th }}$ week or participant no longer intends to continue the smoking cessation program; (8) sponsor cannot continue the trial; and (9) any other cause in which researchers decide discontinuation is appropriate.

\section{Sample Size}

The primary endpoint will be the CAR from weeks 9-24. We estimated conservatively $52.8 \%$ in the CASC treatment group and $40.8 \%$ in the control group as the primary endpoint (CARs from weeks 9-24) based on the survey of smoking cessation [22]. Regarding the effect size, we referred data from a clinical trial of maintenance therapy with varenicline [7], which indicated that 24 weeks of varenicline therapy showed $12 \%$ of additional abstinence rate than placebo in the total population. Thus, we estimated that 24 weeks of the CASC intervention could obtain additional effect of $12 \%$ on abstinence rate compared with the control group. A sample size of 290 in each treatment group will have $80 \%$ power using a chi-square test with a 2-sided significance level of .05 (nQuery Advanced 8.2.1).

\section{Statistical Analysis}

All statistical analyses will be performed according to the intention-to-treat principle and using 2-sided at a .05 significance level. The baseline characteristics will be described by the mean and SD, median and quantiles (continuous variables), or proportion (categorical variables). We will examine the outcomes using the full analysis set. For the primary outcome, we will compare CARs from weeks 9-24 comparing the CASC treatment group and the control group using a logistic regression model adjusted for prescribed smoking cessation medications. In addition, secondary outcomes at each defined period will be evaluated with logistic regression analysis adjusted for appropriate covariates between the groups. We will provide descriptive statistics, odds ratios, $95 \%$ CIs, and $P$ values for each outcome. A statistical analysis plan detailing all statistical computations will be completed prior to the lock of the database.
SAS statistical software, version 9.4 or upper version (SAS Institute Inc), will be used for all the analyses.

\section{Results}

A total of 580 participants with nicotine dependence will be recruited from October 2017 to September 2018 or until recruitment is complete. The final 52-week follow-up will be completed in October 2019. We expect that all trial results will be available by the end of 2019 .

\section{Discussion}

This is the first randomized controlled trial to evaluate the long-term efficacy of CASC, a smoking cessation support app for participants, which also includes a Web-based software for doctors and a mobile $\mathrm{CO}$ checker. We expect that participants in the CASC treatment group, in addition to a 12-week standard smoking cessation program, will exhibit a significantly higher CAR from weeks 9-24 than participants in the control app group.

In recent years, several medical apps for smartphones have shown better clinical outcomes compared with conventional treatment [10-12]. These smartphone apps have also been approved by the US Food and Drug Administration being used in daily clinical practice. In terms of smoking cessation, individuals who used CASC smartphone app without a mobile CO checker achieved $63 \%$ of the CAR from weeks 9-24 compared with those who did not use the app (historical control) [15]. Clickotine, another smartphone app for smoking cessation, also reached $30 \%$ of the abstinence rate at 8 weeks [23]. However, it remains unclear whether CASC including a mobile $\mathrm{CO}$ checker (which provides exhaled $\mathrm{CO}$ concentrations for the user), evidence-based behavioral support, education, and counseling programs for smoking cessation is effective for maintaining long-term abstinence rates in patients with nicotine dependence.

We developed and used a mobile Internet of Things (IoT) device to measure an exhaled $\mathrm{CO}$ concentration level in this trial. A level of exhaled CO concentration is a useful biomarker for patients with nicotine dependence [5]. It helps them understand how much harmful $\mathrm{CO}$ is accumulated in their body and how steadily the harm is decreasing after smoking cessation. They might feel guilty for a high exhaled CO concentration level and try to start quitting, and in another case, they could be encouraged to keep quitting by seeing the decline of their $\mathrm{CO}$ level [24]. These experiences could continuously motivate them and improve adherence to the standard smoking cessation program [25].

There are several benefits to including smartphone apps with an IoT device to clinical settings. First, a mobile app covers treatment gaps between the outpatient clinic visits. It is beneficial to continuously contact participants and keep providing effective treatment programs, which could be one of the most important points for the program success $[10,13]$. Second, medical IoT devices enable us to easily gather biometric information remotely. Patients no longer need to access their outpatient clinics for testing; this might improve patients' total adherence to the treatment program and could contribute to 
construct and promote "telemedicine." Third, the total development costs of the app are much lower than that of medical drugs or medical devices. These apps are highly cost-effective and might reduce burgeoning medical costs [3].

Improving nicotine dependence treatment programs with therapeutic smartphone apps and IoT devices is important in terms of preventing chronic obstructive pulmonary disease, cardio- and cerebrovascular diseases, and malignant tumors. In addition, these mobile apps and devices can contribute to preventing secondhand smoke, which kills approximately 15,000 people every year in Japan [3]. In addition, our device can be a pioneer of the stand-alone programmed medical device with better treatment outcomes. Improving medical outcomes with mobile apps and devices is challenging; however, it can become a highly cost-effective treatment option in the near future. We believe that CASC, which is a combination of a smartphone app, a Web-based patient management software, and a mobile CO checker, will be able to improve the CAR, decrease smoking prevalence and passive smoking, and reduce future total medical costs, while preventing smoking-related diseases in the world.

\section{Acknowledgments}

The authors thank all participants and staff involved in this trial. They also thank Dr Tomoko Betsuyaku for her outstanding support of us and this trial. Furthermore, the authors thank Editage (Tokyo, Japan) for English language editing.

\section{Conflicts of Interest}

This trial is supported by CureApp, Inc. AN has received consulting fees from CureApp, Inc. HT and KF have received honoraria from CureApp, Inc. TM is an employee of CureApp, Inc. KS and SS are the founders and shareholders of CureApp, Inc. and patent holders of the CASC. EH has a consultation contract as a biostatistician with CureApp, Inc. KM has nothing to disclose.

\section{References}

1. Ikeda N, Inoue M, Iso H, Ikeda S, Satoh T, Noda M, et al. Adult mortality attributable to preventable risk factors for non-communicable diseases and injuries in Japan: a comparative risk assessment. PLoS Med 2012 Jan;9(1):e1001160 [FREE Full text] [doi: 10.1371/journal.pmed.1001160] [Medline: 22291576]

2. Ikeda N, Saito E, Kondo N, Inoue M, Ikeda S, Satoh T, et al. What has made the population of Japan healthy? Lancet 2011 Sep 17;378(9796):1094-1105. [doi: 10.1016/S0140-6736(11)61055-6] [Medline: 21885105]

3. Nakamura M. Promoting Tobacco Control for Preventing Passive Smoking: Summary Report (Japanese). 2016. URL: https://mhlw-grants.niph.go.jp/niph/search/NIDD02.do?resrchNum=201608009A [accessed 2018-12-07] [WebCite Cache ID 74U5DtNpT]

4. Igarashi A, Takuma H, Fukuda T, Tsutani K. Cost-utility analysis of varenicline, an oral smoking-cessation drug, in Japan. Pharmacoeconomics 2009;27(3):247-261. [doi: 10.2165/00019053-200927030-00007] [Medline: 19354344]

5. The Standard Procedure Book for Smoking Cessation, 6th edition (Japanese). 2014 Apr. URL: https://www.jrs.or.jp/uploads/ uploads/files/information/non-smoking 06.pdf [accessed 2018-12-10] [WebCite Cache ID 74ZVOvjoU]

6. Ministry of Health, Labour and Welfare (Japan). 2017. Report on the Efficacy of Smoking Cessation Treatment Based on Nicotine Dependency Management Fee URL: https://www.mhlw.go.jp/file/05-Shingikai-12404000-Hokenkyoku-Iryouka/ 0000192293.pdf [accessed 2018-12-07] [WebCite Cache ID 74U5NcjPx]

7. Tonstad S, Tønnesen P, Hajek P, Williams KE, Billing CB, Reeves KR, Varenicline Phase 3 Study Group. Effect of maintenance therapy with varenicline on smoking cessation: a randomized controlled trial. JAMA 2006 Jul 05;296(1):64-71. [doi: $10.1001 /$ jama.296.1.64] [Medline: 16820548 ]

8. Nakamura M, Oshima A, Fujimoto Y, Maruyama N, Ishibashi T, Reeves KR. Efficacy and tolerability of varenicline, an alpha4beta2 nicotinic acetylcholine receptor partial agonist, in a 12-week, randomized, placebo-controlled, dose-response study with 40-week follow-up for smoking cessation in Japanese smokers. Clin Ther 2007 Jun;29(6):1040-1056. [doi: 10.1016/j.clinthera.2007.06.012] [Medline: 17692720]

9. Quinn CC, Clough SS, Minor JM, Lender D, Okafor MC, Gruber-Baldini A. WellDoc mobile diabetes management randomized controlled trial: change in clinical and behavioral outcomes and patient and physician satisfaction. Diabetes Technol Ther 2008 Jun;10(3):160-168. [doi: 10.1089/dia.2008.0283] [Medline: 18473689]

10. Quinn CC, Shardell MD, Terrin ML, Barr EA, Ballew SH, Gruber-Baldini AL. Cluster-randomized trial of a mobile phone personalized behavioral intervention for blood glucose control. Diabetes Care 2011 Sep;34(9):1934-1942 [FREE Full text] [doi: 10.2337/dc11-0366] [Medline: 21788632]

11. Ebert DD, Zarski A, Christensen H, Stikkelbroek Y, Cuijpers P, Berking M, et al. Internet and computer-based cognitive behavioral therapy for anxiety and depression in youth: a meta-analysis of randomized controlled outcome trials. PLoS One 2015;10(3):e0119895 [FREE Full text] [doi: 10.1371/journal.pone.0119895] [Medline: 25786025]

12. Bricker JB, Mull KE, Kientz JA, Vilardaga R, Mercer LD, Akioka KJ, et al. Randomized, controlled pilot trial of a smartphone app for smoking cessation using acceptance and commitment therapy. Drug Alcohol Depend 2014 Oct 1;143:87-94. [doi: 10.1016/j.drugalcdep.2014.07.006] [Medline: 25085225] 
13. Free C, Knight R, Robertson S, Whittaker R, Edwards P, Zhou W, et al. Smoking cessation support delivered via mobile phone text messaging (txt2stop): a single-blind, randomised trial. Lancet $2011 \mathrm{Jul}$ 2;378(9785):49-55 [FREE Full text] [doi: 10.1016/S0140-6736(11)60701-0] [Medline: 21722952]

14. Masaki K. Preliminary Report of a Smartphone Application "CureApp" for Smoking Cessation. 2017 Presented at: American Thoracic Society; 05/24/2017; Washington, USA.

15. Masaki K, Tateno H, Kameyama N, Morino E, Watanabe R, Sekine K, et al. Impact of a Novel Smartphone Application "CureApp Smoking Cessation" on Nicotine Dependence: Prospective Single-arm Interventional Pilot Study. JMIR mHealth uHealth 2018. [doi: 10.2196/preprints.12694] [Medline: 30181109]

16. Masaki K, Tateno H, Morino E. Multi-center, Single-Arm Study to Evaluate Efficacy of Smartphone Application "CureApp" for Smoking Cessation: Interium Report. 2017 Presented at: The 634th Kanto Reginal Meeting, Japanese Society of Internal Medicine; 07/09/2017; Tokyo, Japan.

17. Masaki K, Tateno H, Kameyama N. A Multi-center, Single-Arm Study to Evaluate Efficacy of Smartphone Application "CureApp" for Smoking Cessation. 2018 Presented at: The Japanese Society of Internal Medicine, Annual Scientific Meeting; April 2018; Kyoto, Japan.

18. West R, Hajek P. Evaluation of the mood and physical symptoms scale (MPSS) to assess cigarette withdrawal. Psychopharmacology (Berl) 2004 Dec;177(1-2):195-199. [doi: 10.1007/s00213-004-1923-6] [Medline: 15179542]

19. Berlin I, Singleton EG, Heishman SJ. Validity of the 12-item French version of the Tobacco Craving Questionnaire in treatment-seeking smokers. Nicotine Tob Res 2010 May;12(5):500-507 [FREE Full text] [doi: 10.1093/ntr/ntq039] [Medline: 20335281]

20. Otani T, Yoshii C, Kano M, Kitada M, Inagaki K, Kurioka N, et al. Validity and reliability of Kano Test for Social Nicotine Dependence. Ann Epidemiol 2009 Nov;19(11):815-822. [doi: 10.1016/j.annepidem.2009.03.016] [Medline: 19457683]

21. Kawakami N, Takatsuka N, Inaba S, Shimizu H. Development of a screening questionnaire for tobacco/nicotine dependence according to ICD-10, DSM-III-R, and DSM-IV. Addict Behav 1999;24(2):155-166. [Medline: 10336098]

22. Ministry of Health, Labour and Welfare (Japan). 2008. Survey on the actual status of smoking cessation rate in medical institutions calculating insurance fee for nicotine dependence management URL: https://www.mhlw.go.jp/shingi/2008/07/ dl/s0709-8k.pdf [accessed 2018-12-07] [WebCite Cache ID 74U5umcPo]

23. Iacoviello BM, Steinerman JR, Klein DB, Silver TL, Berger AG, Luo SX, et al. Clickotine, A Personalized Smartphone App for Smoking Cessation: Initial Evaluation. JMIR Mhealth Uhealth 2017 Apr 25;5(4):e56 [FREE Full text] [doi: 10.2196/mhealth.7226] [Medline: 28442453]

24. Herbeć A, Perski O, Shahab L, West R. Smokers' Views on Personal Carbon Monoxide Monitors, Associated Apps, and Their Use: An Interview and Think-Aloud Study. Int J Environ Res Public Health 2018 Dec 07;15(2) [FREE Full text] [doi: 10.3390/ijerph15020288] [Medline: 29414907]

25. Jamrozik K, Vessey M, Fowler G, Wald N, Parker G, Van Vunakis H. Controlled trial of three different antismoking interventions in general practice. Br Med J (Clin Res Ed) 1984 May 19;288(6429):1499-1503 [FREE Full text] [Medline: $\underline{6426618]}$

\section{Abbreviations}

AI: artificial intelligence

CAR: continuous abstinence rate

CASC: CureApp Smoking Cessation

CO: carbon monoxide

FTCQ-12: 12-item French version of the Tobacco Craving Questionnaire

IoT: Internet of Things

KTSND: Kano Test for Social Nicotine Dependence

MPSS: Mood and Physical Symptoms Scale

Edited by G Eysenbach; submitted 25.09.18; peer-reviewed by R Vilardaga, N Watson, J Steinerman; comments to author 10.11.18;
revised version received 24.11.18; accepted 24.11.18; published 11.02.19
Please cite as:
Nomura A, Tateno H, Masaki K, Muto T, Suzuki S, Satake K, Hida E, Fukunaga K
A Novel Smoking Cessation Smartphone App Integrated With a Mobile Carbon Monoxide Checker for Smoking Cessation Treatment:
Protocol for a Randomized Controlled Trial
JMIR Res Protoc 2019;8(2):e12252
URL: http://www.researchprotocols.org/2019/2/e12252/
doi: $10.2196 / 12252$
PMID: $\underline{30741641}$


(C)Akihiro Nomura, Hiroki Tateno, Katsunori Masaki, Tomoyasu Muto, Shin Suzuki, Kohta Satake, Eisuke Hida, Koichi Fukunaga. Originally published in JMIR Research Protocols (http://www.researchprotocols.org), 11.02.2019. This is an open-access article distributed under the terms of the Creative Commons Attribution License (https://creativecommons.org/licenses/by/4.0/), which permits unrestricted use, distribution, and reproduction in any medium, provided the original work, first published in JMIR Research Protocols, is properly cited. The complete bibliographic information, a link to the original publication on http://www.researchprotocols.org, as well as this copyright and license information must be included. 